\title{
Cerebral Gas Embolism - A Serious Complication of $\mathrm{CO}_{2}$ Angiography
}

\author{
SUMIT KAPOOR ${ }^{1, *}$, Nariman Nezami ${ }^{1}$, Ross Mazo $^{1}$, Jyotsana Thakkar $^{2}$ \\ ${ }^{1}$ United States, Jacobi Medical Center, NY \\ ${ }^{2}$ North Shore LIJ Hospital \\ *Corresponding author: drkapoorsumit@gmail.com
}

Received June 12, 2015; Revised June 19, 2015; Accepted June 25, 2015

\begin{abstract}
Carbon dioxide angiography is routinely used for vascular imaging and endovascular procedures. It is very useful in patients with allergy to iodinated contrast agents or with poor renal function. It is relatively safe to use. We describe first case of cerebral gas embolism leading to multiple bilateral ischemic strokes in a patient receiving carbon dioxide angiography for workup of thrombosed arm arteriovenous fistula.
\end{abstract}

Keywords: thrombosed, arteriovenous, digital subtraction angiography, stroke

Cite This Article: SUMIT KAPOOR, Nariman Nezami, Ross Mazo, and Jyotsana Thakkar, "Cerebral Gas Embolism - A Serious Complication of $\mathrm{CO}_{2}$ Angiography.” American Journal of Medical Case Reports, vol. 3, no. 8 (2015): 227-230. doi: 10.12691/ajmcr-3-8-2.

\section{Introduction}

Carbon Dioxide $\left(\mathrm{CO}_{2}\right)$ angiography has become a widely used modality for vascular imaging and endovascular procedures [1,2]. Its applications include digital subtraction angiography, aortography, venography, renal arteriography, visceral angiography, transcatheter embolization, angioplasty and stenting, endovascular abdominal aortic aneurysm repair and TIPS placement [1-8]. It is particularly useful in patients who are hypersensitive to iodinated contrast or have compromised renal function [9]. We report the first case of $\mathrm{CO}_{2}$ gas embolism causing multiple diffuse bilateral strokes in a patient undergoing $\mathrm{CO}_{2}$ angiogram for thrombosed $\mathrm{AV}$ fistula.

\section{Case Description}

A 54 year old Male with past medical history of Diabetes Mellitus, Hypertension, Coronary artery disease and kidney transplant in 2007 for End stage renal disease, was admitted to a vascular center for outpatient $\mathrm{CO}_{2}$ angiography for signs and symptoms concerning for Right upper extremity ischemia from thrombosed right arm Arteriovenous fistula. Immediately post procedure,his mental status deteriorated and developed focal neurologic deficits (Left upper extremity weakness and left facial droop). He was intubated for airway protection due to poor mental status and was transferred to our hospital for hyperbaric oxygen therapy for possible $\mathrm{CO}_{2}$ cerebral gas embolism. Head CT did not reveal acute stroke. MRI brain showed multiple bilateral white matter infarcts in frontal, parietal and occipital lobes (MR images 1 to 5). Transesophageal echocardiography showed no cardiac thrombus or intracardiac shunts. His ICU course was complicated by Acute respiratory distress syndrome, septic shock, seizures, pneumonia and renal failure requiring renal replacement therapy. There was no meaningful improvement in his mental status and underwent tracheostomy and PEG tube placement as per family wishes and transferred to nursing home.

\section{Discussion}

$\mathrm{CO}_{2}$ is widely used as an intravascular contrast agent for imaging of the arterial and venous circulations. It is particularly useful in patients with renal insufficiency or those who have allergy to iodinated contrast agents. It is a colorless and odorless gas which is 20 times more soluble in blood than oxygen. It forms bubbles in the vessels which completely dissolve in 2-3 minutes. Therefore, repeated injections of $\mathrm{CO}_{2}$ are given 2-3 minutes apart so that it does not accumulate and form large bubbles in vessels. It has low viscosity leading to better filling and visualization of the collateral vessels in the vascular occlusive diseases. Adverse effects reported due to $\mathrm{CO}_{2}$ angiography are related to the dose and air contamination. Pain at the injection site during peripheral venography and nausea and abdominal pain during celiac arteriography have been reported $[2,10] . \mathrm{CO}_{2}$ trapped in aortic aneurysm may lead to colonic ischemia $[2,10]$. Injection of excessive volumes of $\mathrm{CO}_{2}$ or air contamination can lead to vapor lock in the pulmonary artery and severe hypotension that persists for 30 seconds and improves with placing the patient in left lateral decubitus or trendelenburg position [2]. There are no absolute contraindications for $\mathrm{CO}_{2}$ angiography but should not be used for imaging of thoracic aorta and arterial vasculature above the diaphragm due to the risk of carotid, spinal and coronary artery gas embolism. It should be avoided in patients with pulmonary hypertension since it increases the pulmonary artery 
pressure and in patients with known intracardiac shunts or pulmonary AV malformations as it may cause paradoxical embolism. We report first case of upper extremity $\mathrm{CO}_{2}$ angiography causing carotid artery gas embolism leading to diffuse bilateral ischemic strokes. In our case, the explanation is that thrombosed AV fistula led to retrograde movement of the $\mathrm{CO}_{2}$ bubbles and entered bilateral cerebral circulation.

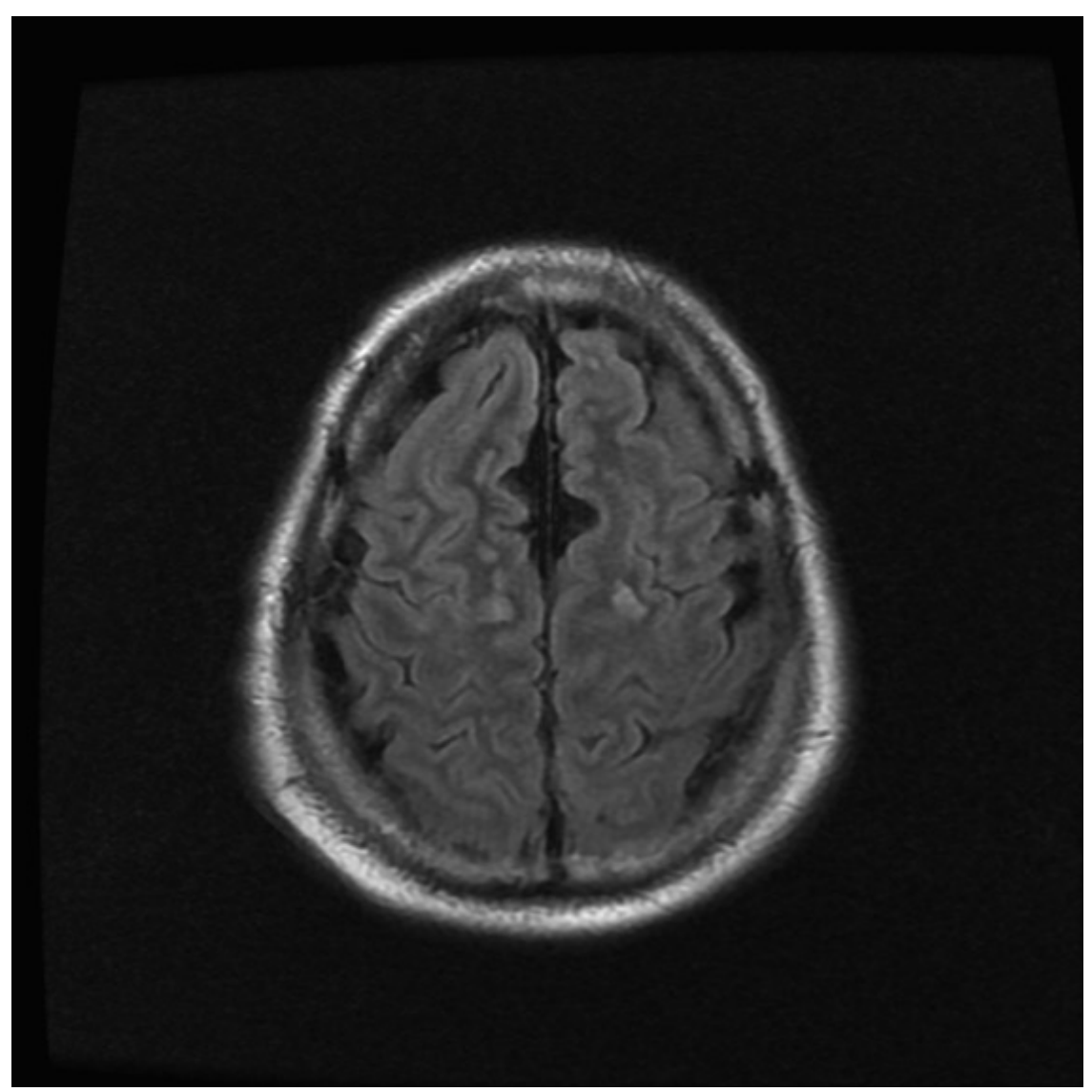

Figure 1. MRI Image 1

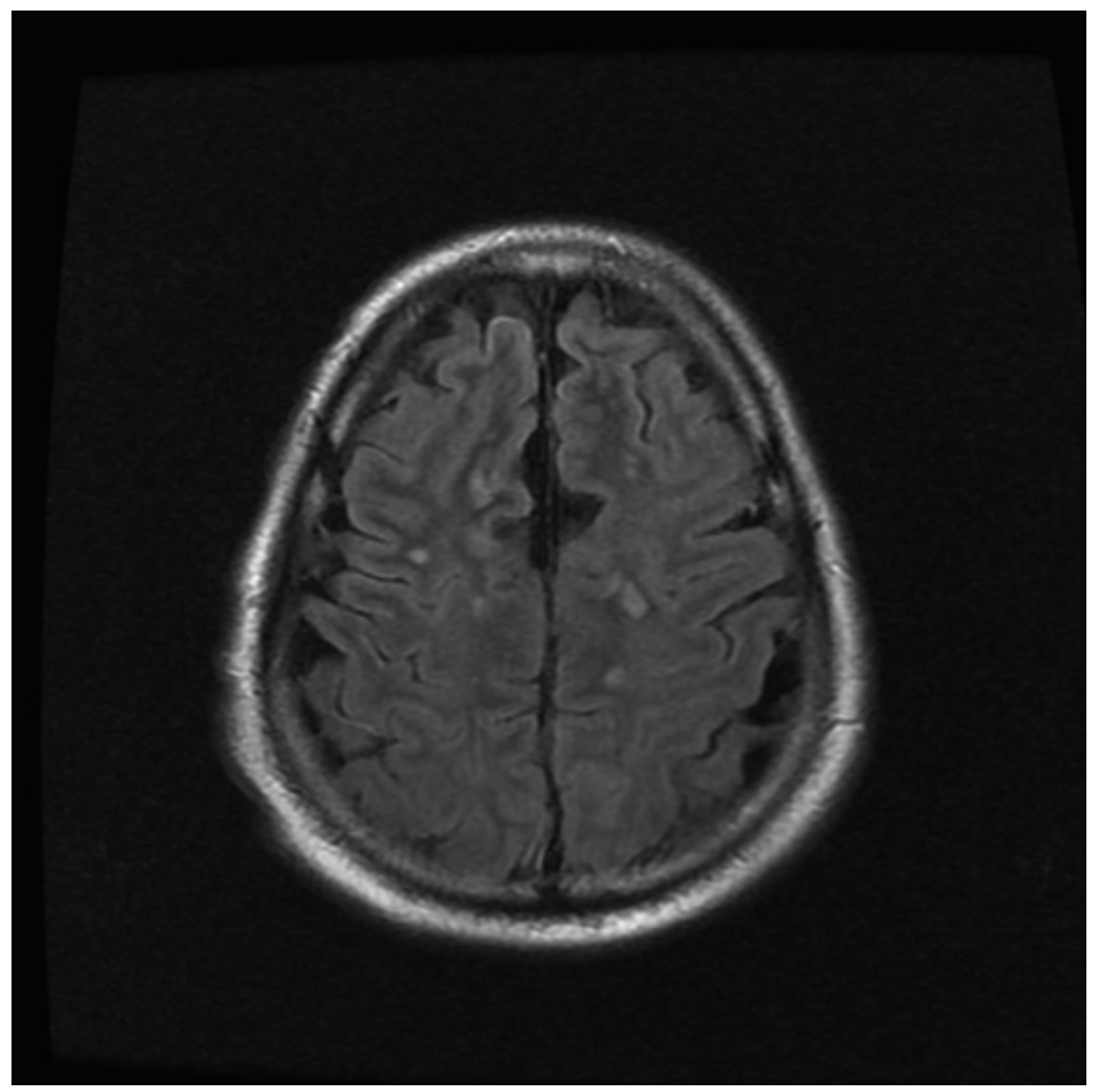

Figure 2. MRI Image 2 


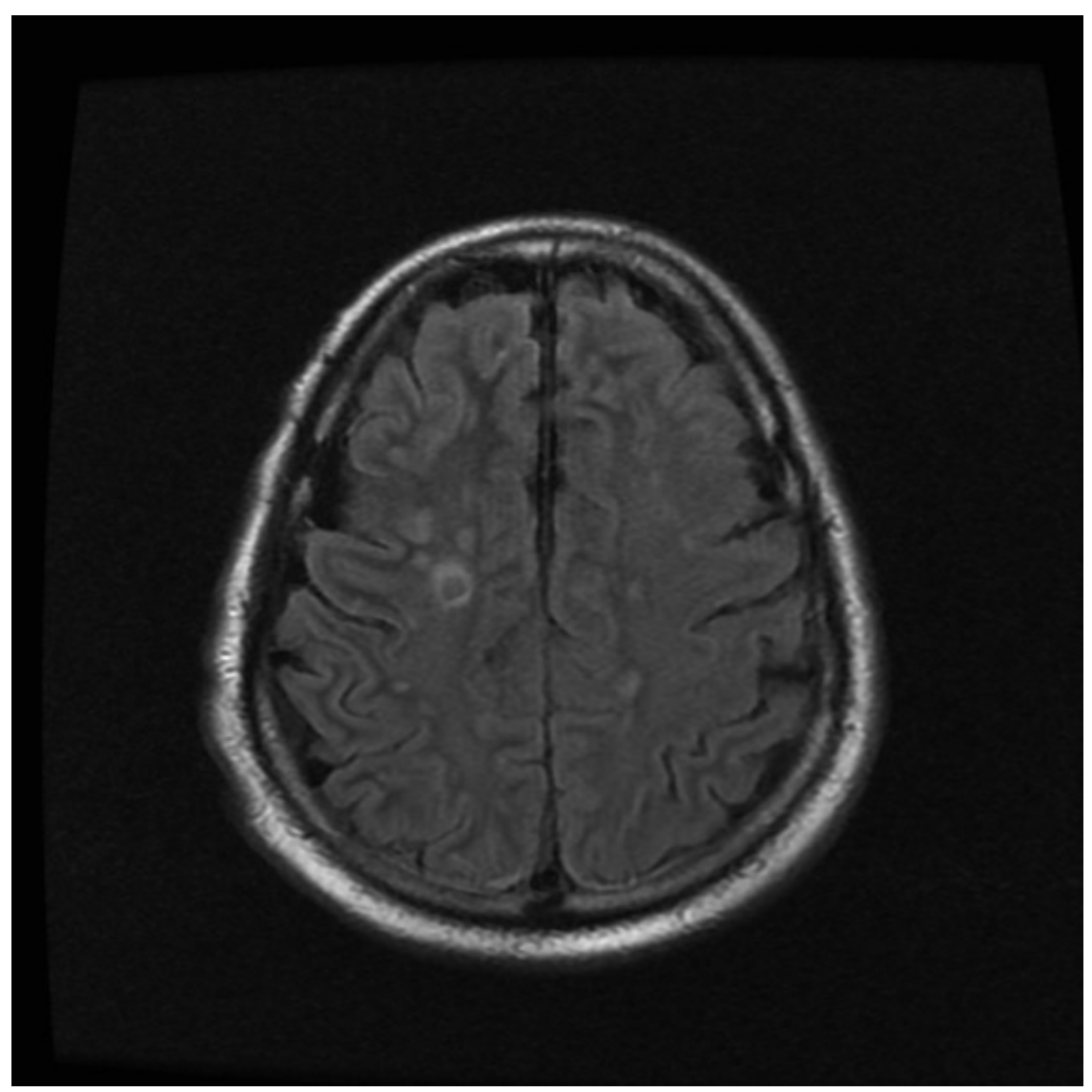

Figure 3. MRI Image 3

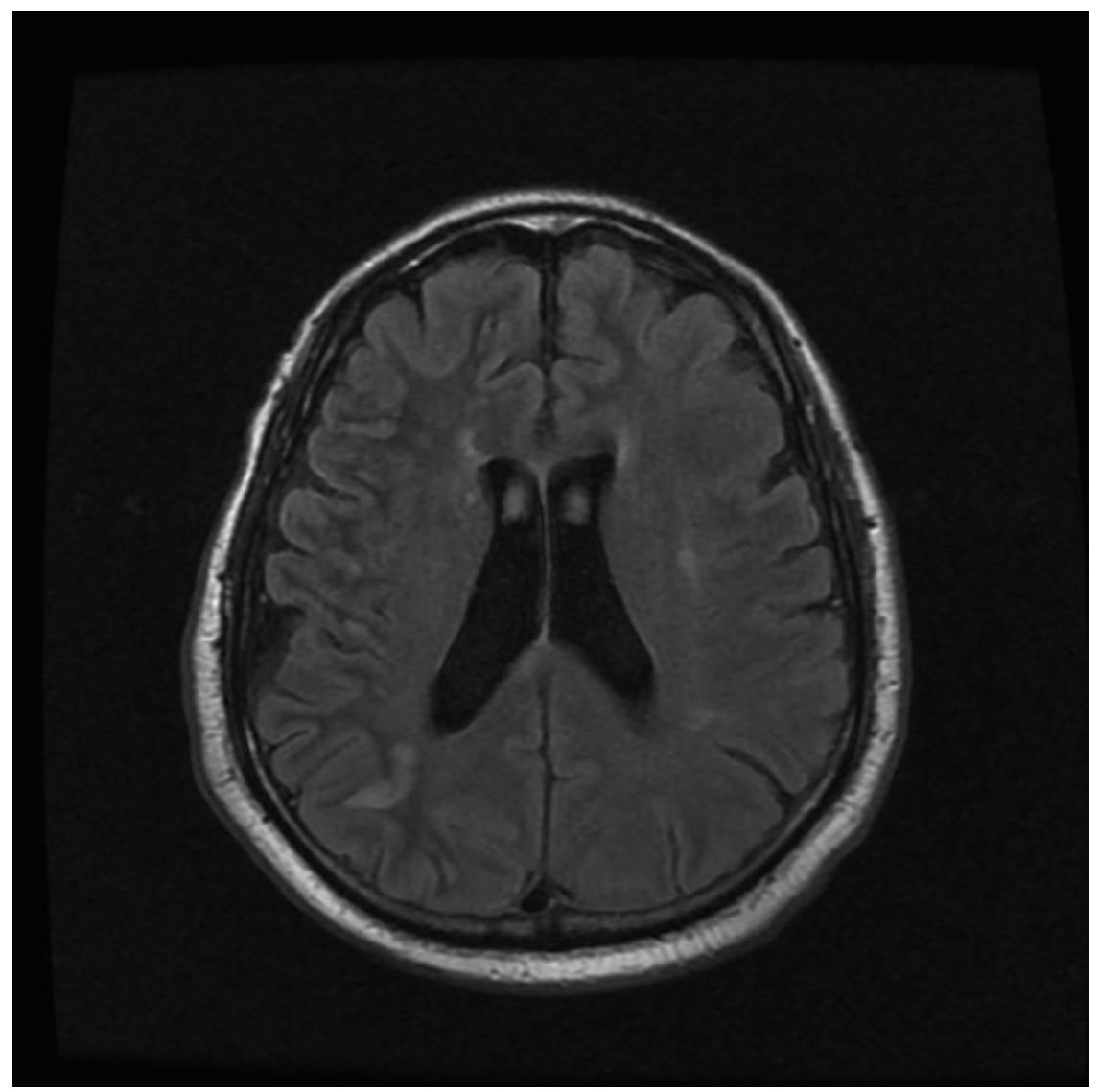

Figure 4. MRI Image 4

\section{Conclusion}

Intensivists should be aware of the reported adverse effects of $\mathrm{CO}_{2}$ angiography since we care for this patient population in our ICUs. We report first case of cerebral 
gas embolism due to $\mathrm{CO}_{2}$ angiography which left patient

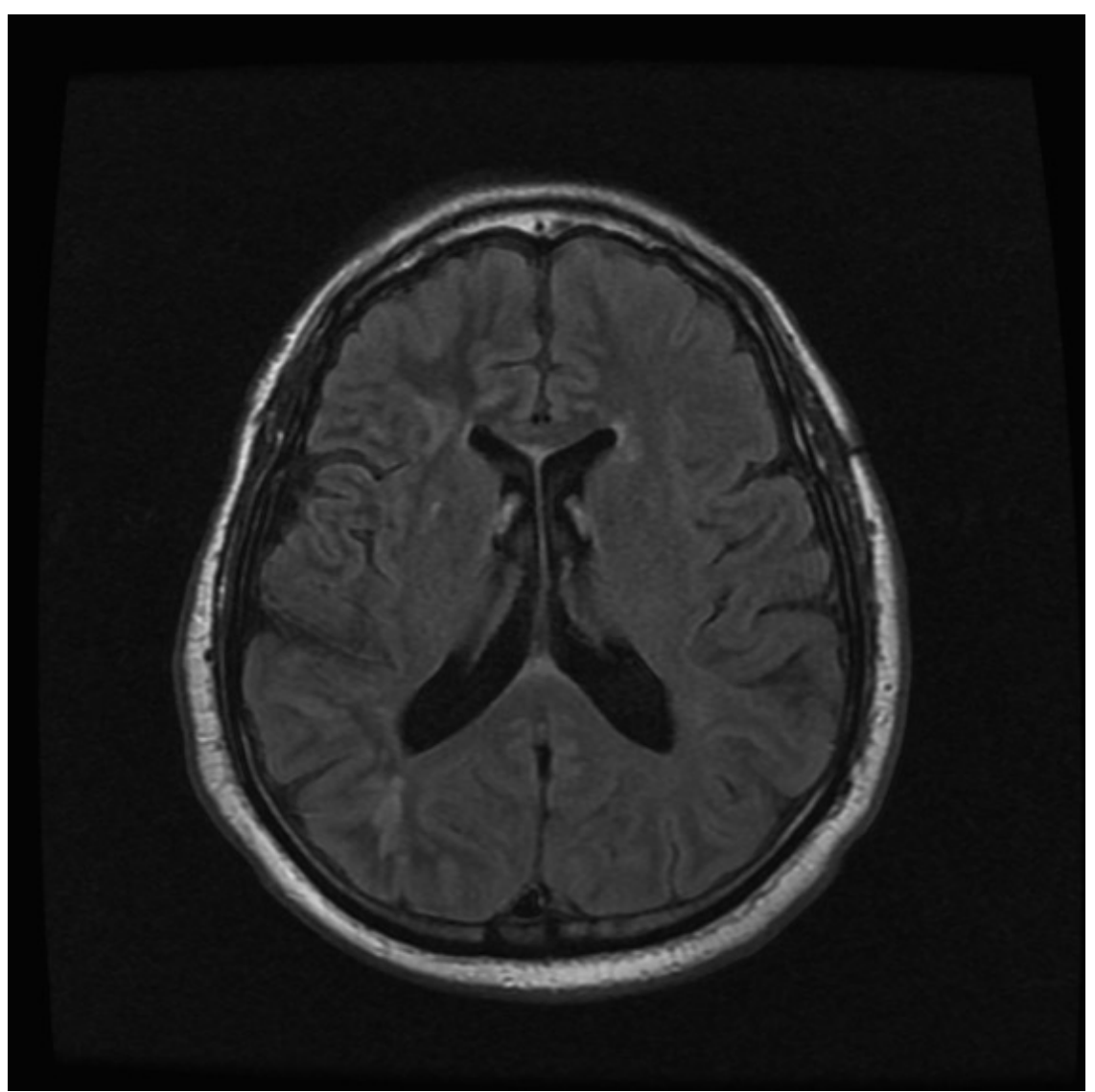

Figure 5. MRI Image 5

\section{References}

[1] Carbon dioxide angiography. Kyung $J$ et al. Informa healthcare; 2007.

[2] Carbon dioxide angiography. Kyung J et al. Medscape. Sept 2013

[3] Carbon dioxide digital subtraction angiography: the practical approach. Caridi JG et al. Tech Vasc Interv Radiol. March 2001; 4(1): 57-65.

[4] Carbon dioxide digital subtraction angiography: expanding applications and technical evolution. Kerns et al. American Journal of Roentgenology. 1995; 164: 735-741.

[5] $\mathrm{CO}_{2}$ angiography. Huber et al. Catheterization and cardiovascular Interventions. Vol 55, Issue 3, pages 398-403.
[6] Renal angiography using carbon dioxide. Beese $R C$ et al. $\mathrm{Br} \mathrm{J}$ Radiol. Jan 2000; 73 (865): 3-6.

[7] Assessment of $\mathrm{CO}_{2}$ angiography in arterial occlusive disease of the lower extremities. Diaz et al. J Vasc Interv Radiol. Feb 2000; 11 (2 pt 1): 163-9.

[8] Catheter-less angiography for endovascular aortic aneurysm repair: A new application of carbon dioxide as a contrast agent. Criado et al. J Vasc Surg. Jul 142008.

[9] Contrast alternatives for iodinated contrast allergy and renal dysfunction: options and limitations. Nadolski et al. J Vasc Surg. Feb 2013; 57 (2): 593-8.

[10] Safety of carbon dioxide digital subtraction angiography. Moos JM et al. Arch Surg. Dec 2011; 146 (12): 1428-32. 